\section{Über eine Fehlerquelle bei der Elementaranalyse von Kohlen, die eine größere Menge kohlensaure Erden enthalten.}

\author{
Von Dr. Knublacce, Köln-Ehrenfeld. \\ (Eingeg. 10./10. 1915.)
}

Uber den Einfluß der kohlensauren Erden in Kohlen und minderwertigen Brennstoffen auf die Bestimmung des Kohlenstoffes und Sauerstoffes durch Flementaranalyse findet sich in der Literatur nichts verzeichnet. Da der dadurch entstehende Fehler recht groß sein kann und dann nicht zu vernachlässigen ist, so teile ich meine Erfahrungen darüber mit, namentlich auch in bezug auf die Heizwertbestimmung durch Elementaranalyse. Stimmt diese mit einer exakten calorimetrischen Bestimmung nicht genügend überein, so ist der Fehler in der Ausführung der Elementaranalyse zu suchen. Neben genauer Bestimmung der Elemente, namentlich auch des Schwefels und Stickstoffs ${ }^{1}$ ), muß ev. auch der durch den Kohlensäuregehalt der mineralischen Stoffe in der Kohle verursachte Fehler berücksichtigt und auf Grund der Kohlensäurebestimmung korrigiert werden.

Verschiedene Beobachter sprechen sich dahin aus, da die calorimetrischen Heizwertbestimmungen mit den aus der Flementaranalyse ermittelten, genügend übercinstimmen - in beiden Fällen natürlich ein pein li ch ge n a u es Arbeiten und rich tige Korrekt u ren vorausgesetzt. Erspart man sich aber bei der calorimetrischen Untersuchung die Korrektur für Schwefelsäure und Salpetersäure oder die Bestimmung des Verbrennungswassers und berechnet dies aus dem durchschnittlichen Wasserstoffgehalt der betreffenden Kohlenart, wic das vorgeschlagen ist, so ergibt ohne Zweifel die exakte Elementaranalyse - selbstredend mit Bestimmung von Schwefel und Stickstoff - zuverlässigere Resultate, und es ist zu betonen, daß3 die Sicherheit durch die hier in Rede stehende Korrektur betreffs Kohlenstoffs und Sauerstoffs bzw. disponiblen Wasserstoffs bei manchen Proben noch erhöht wird.

Es ist auffallend, daß man häufig eine gewisse Scheu vor der Elementaranalyse beobachtet, und da $ß$ zu deren Ausführung mit besonderer Ängstlichkeit geschritten wird, während diese Untersuchung doch zu den elegantesten Experimenten zählt Allerdings dürfte es schon manchem Analytiker vorgekom men sein, da $B$ die Summe der einzelnen Bestimmungen sich schlecht fügt zu, ,dem Rest als $O^{*}$, und die Untersuchung sogar mehrmals wiederholt werden muß. Zur Klärung der Frage einer genaueren Kohlenstoff- und Sauerstoffbestimmung in manchen Kohlen soll diese Arbeit mit beitragen.

Da man bei der calorimetrischen Untersuchung nicht auch ,auf 100" zu rechnen braucht, so macht sich da ein Fehler bei der ersten Bestimmung nicht so leicht bemerkbar. In nicht durchaus geübter Hand und bei nicht genauen Korrekturen und nicht durchaus zuverlässiger Bestimmung des Wasserwertes des Calorimeters kommen hier leicht größere Fehler vor als bei der Elementaranalyse, die jeder Chemiker schon während des Studiums ausführt. Ủber Irrtümer und Fehler bei der Heizwertbestimmung von Brennstoffen macht auch $\mathrm{L}$ a $\mathrm{n} \mathrm{g}$ b e in ${ }^{2}$ ) interessante Mitteilungen, dessen reiche Erfahrung in bezug auf calorimetrische Untersuchungen niemand erreicht baben dürfte.

Ist es auch bei der immer sicherer ausgebildeten calorimetrischen Methode erklärlich, daß diese namentlich von Speziallahoratorien, welche darin die nötige Utbung und Sicherheit besitzen, der Elementaranalyse vorgezogen wird, so ist letztere doch für die Heizwertbestimmung keineswegs verlassen, und es fehlt auch noch z u rzeit nicht an Stimmen, welche dieser das Wort reden, zumal die Zeitersparnis bei der calorimetrischen Untersuchung namentlich da, wo dieselbe nur von Zeit zu Zeit vorkommt, überschätzt wird. Es wird meist überseben, daß die Bestimmungen für

1) Über Bestimmung des $N$ in Kohlen s. Kn u bla u c h, J. f. Gasbel. 55, 713 [1912] und Angew. Chem. 26. I, 425 [1913]. Hier kommen große Fehler vor, wie auch aus dem oft so hohen Säureverbrauch beim blinden Versuch zu ersehen ist.

2) Rückblicke auf die Tätigkeit des öffentlichen chemischen SpezialLaboratoriums für calorimetrische Untersuchungen von Dr. $\mathrm{L}$ a $\mathrm{n} g$ b e i.n in Niederlößnitz bei Dresden (Niederlößnitz, Sommer 1906). die verschiedenen Korrekturen den Zeitaufwand für die beiden calorimetrischen Versuche wesentlich crhöhen, so die Ermittlung der gebildeten Salpctersäure und Schwefelsäure und namentlich des Verbrennungswassers, durch Elementaranalyse. Wird nun diese doch ausgeführt für die Wasserstoff bestimmung (was doch bei Brennmaterialien von unbekannter Herkunft und hohem Aschengehalt erst recht nötig ist), so ist der C-Gehalt leicht mitbestimmt, und dem Zeitaufwande für den calorimetrischen Versuch, einschliel3lich Bestimmung von Salpetersäure und Schwefelsäure steht dann nur die für die Wägungen des Kaliapparates und äie Ermittlung von $\mathrm{S}$ und $\mathrm{N}$ und ev. der $\mathrm{CO}_{2}$ nötige Zeit gegenüber, Bestimmungen, die in geübter Hand wenig Zeit erfordern, da während des Glühens und Zersetzens andere Arbeiten erledigt werden könneu.

Daß3 diese Frage auch Kreise beschäftigt, die diese Untersuchungen fortwährend ausführen, und das auch noch in neuerer Zeit, beweist u. a. eine Verhandlung der Chemikerkommission des Vereins deutscher Fisenhüttenleute am 23./3. 1912 (Bericht Nr.9). Hier hebt Wen zelius zunächst die Zuverlässigkeit der calorimetrischen Bestimmung in der Hand e in es g e ü b t e n Chemikers hervor und bemerkt, da $B$ mehr als vier an einem Tage nicht zu erlcdigen seien, sämtliche Bestimmungen müßten doppelt ausgeführt werden; auch sei die Anschaffung ziemlich kostspielig, man müsse für die $\mathbf{M}$ a h $\mathrm{l}$ e r sche Bombe (innen nicht platiniert, sondern emailliert) etwa $1500 \mathrm{M}$ rechnen. W. wirft die Frage auf, ob es nicht praktischer wäre (da die Bestimmung des Heizwertes einer Kohle zwei Versuche in der Bombe und cine Elementaranalyse für die Wasserbestimmung nötig macht), die Elementaranalyse vollständig durchzuführen, da man so ganz von dem calorimetrischen Versuche mit der Bombe absehen und den Heizwert nach der $\mathrm{D}$ u l o $\mathrm{n}$ g e schen Formel ermitteln könne. W. führt dann 22 Kohlenproben an, mit welchen er die Verbrennungswärme mit dern Calorimeter und nach der Elementaranalyse bestimmte. Hiervon weichen 16 Proben ganz unwesentlich ab $(0,1$ bis $0,5 \%$ ), und die grölßte Differenz bei den übrigen 6 Proben beträgt $1,85 \%{ }^{\circ}$ Bei Berücksichtigung der hier in Rede stehenden Korrektur bezüglich des $\mathrm{CO}_{2}$ - Gehaltes in der Kohle dürfte häufig eine noch bessere Ưbereinstimmung erreicht werden.

Kalk und $\mathrm{Mg}$ der Kohle sind häufig zu einem nicht unbedeutenden Teil an Kohlensäure gebunden. Dieselbe entweicht aus dem $\mathrm{CaCO}_{3}$ bei etwas über $600^{\circ}$ (aus der $\mathrm{MgCO}_{3}$ schon bei über $300^{\circ}$ ). Da die Kohle bei der Elementaranalyse im Sauerstoffstrom verbrannt wird, so ist die Temperatur, abgesehen von der äußeren Erhitzung des Rohres, höher, so daß3 diese $\mathrm{CO}_{2}$ im Kaliapparat mit zur Wägung kommt. Dadurch entstehen folgende Fehler:

1. Der C-Gehalt fällt stets um $3 / 11$ der an diese Basen gebundenen $\mathrm{CO}_{2}$ zu hoch aus, was recht erheblich sein kann (bei Probe 1 z. B. $1,00 \%$; S 493).

2. Falls als Asche der bei der Elementaranalyse im Schiffchen zurückbleibende Rückstand angenommen wirà, was sich allerdings nur bei hohem Aschengehalt empfiehlt (s. unten 21,95 und $68,70 \%$ ), so berechnet sich der O-Gehalt um die $\mathrm{CO}_{2}$ der kohlensauren Erden zu boch und um 3/1 dieser $\mathrm{CO}_{2} \mathrm{zu}$ niccirig, so daß $8 / 11$ der $\mathrm{CO}_{2}$ von $\mathrm{O} \mathrm{zu}$ subtrahieren ist; bei Probe Nr. I beträgt $\mathrm{z}$. B. die Differenz $93,20 \times 0,04=3,73 \times 8 / 11=2,71 \%$ O, (S. 493).

Ferner betrug die Asche der Kohle Nr. 3 nach dem Glühen über dem Gebläse (entsprechend im Schiffchen bei der Elementaranalyse) a) $11,05 \%$ und nach dem Erhitzen mit kohlensaurem Ammoniak (s. unten ad 3) b) $12,20 \%$, Differenz $=1,15 \%$. Vom O-Gehalt der Elementaranalyse nach a) berechnet, ist somit $1,15 \times 8 / 11==0,84 \% \mathrm{zu}$ subtrahieren. Genügend überein stimmt daınit die Berechnung aus dem $\mathrm{CO}_{2}$ - Gehalt derselben Probe $=27,8 \times 0,04 \times 8 / 11$ gleich $0,81 \%$, die vom $\mathrm{O}$ zu subtrahieren sind, um das Mehr durch die Kohlensäure und den Ausfall an C durch dieselbe auszugleichen. (Bei der Aschenbestimmung in der Muffel dürfte alle $\mathrm{CO}_{2}$ ausgetrieben werden, und sich der Fehler wie in diesen beiden Beispielen stellen. Nach dem nachstehend unter ad 3) Gesagten kann durch kohlensaures Arnmoniak der Fehler ausgeglichen und der $\mathrm{O}$-Gehalt richtig berechnet werden.) 
3. Bei Bestimmung der Asche im Porzellantiegel ${ }^{3}$ ) über dem Bunsenbrenner wird je nach Temperatur und Zeit mehr oder weniger $\mathrm{CO}_{2}$ ausgetrieben (in der Muffel mehr, ev, alle $\mathrm{CO}_{2}$ ), so daß cier $\mathrm{O}$-Gehalt dementsprechend um eine $\mathrm{u} \mathrm{n}$ be k a n $n$ te Größe zu hoch gefunden wird, und so eine Korrektur ohne weiteres nicht möglich ist. Es empfiehlt sich deshalb, v o r der Berechnung des $\mathrm{O}$ als Differenz oie Asche mit etwas kohlensaurem Ammoniak zu erhitzen, zuletzt ziemlich stark mit direkter Flamme, aber nicht bis zum Glühen.

So ergab bei Kohle Nr. 3 die Aschenbestimmung mit dem Bunsenbrenner 11,80\% und nach Behandeln mit kohlensaurem Ammoniak 12,20\%. Diese Differenz von $0,40 \%$ kann natürlich je nach Temperatur und Zeit s e hr ver $\mathrm{s}$ c h i e d e $\mathrm{n}$ sein, und die Berechnung des $\mathrm{O}$ aus der Asche direkt, wie das üblich ist, gibt so Fehler von verschiedener Größc. Beim Behandeln der Asche mit kohlensaurem Ammoniak ist eine Korrektur nach dieser Richtung somit nicht nötig man hat nur von dem durch Elementaranalyse gefundenen $\mathrm{C}$ den der Kohlensäure der Kohle äquivalenten $\mathrm{C}\left(3 / 11 \mathrm{CO}_{2}\right)$ zu subtrahieren und dieselbe Größe dem $O$ zu adaieren. (Berechnung s. weiter unten.)

Umgekehrt könnte man auch die $\mathrm{CO}_{2}$ der $\wedge$ sche durch Glühen vor dem Gebläse vollständig austreiben und von dem danu berechneten $\mathrm{O}$ die $\mathrm{CO}_{2}$ der Kohle und vom $\mathrm{C}^{3} / 1$ dieser $\mathrm{CO}_{2}$ subtrahieren.

Da ciie Zusammensetzung der Asche naturgemäß sehr verschieden ist, so kann man aus deren Menge natürlich nicht auf die Menge $\mathrm{CO}_{2}$ schließen, aber bei geringem Aschengehalt kann natürlich die Korrektur unterbleiben, denn anch im ungünstigsten Falle ist dann der durch $\mathrm{CO}_{2}$ verursachte Fehler ohne Bedeutung.

Nachstehend ist die Kohlensäure einer Reihe Kohlen und minderwertiger Materialien von verschiedener Herkunft und mit sehr verschiedenem Aschengehalt aus je $5 \mathrm{~g}$ in ccm bei $0^{\circ}$ aufgeführt und zugleich der Aschengehalt, woraus auch zu ersehen ist, daß Kohlensäure und Asche keineswegs in einem bestimmten Verhältnis stehen. So gibt die Gaskohle $\mathrm{Nr}$. 3 bei $11,8 \%$ sche $27,8 \mathrm{~cm} \mathrm{CO} \mathrm{CO}_{2}$ und die Waschberge Nr. 4 bei der sechsfachen Menge Asche weniger $\mathrm{CO}_{2}$. Fbenso gibt die Kohle 10 mit sehr hohem Aschengehalt nur 5,24 ccm Kohlensäure, Probe 1 mit weniger als zwei Drittel Asche aber 93,2 ccm $\mathrm{CO}_{2}$. Der $\mathrm{CO}_{2}$-Gehalt dieser 12 Proben schwankt von 93,20 bis $1,18 \mathrm{ccm} \mathrm{CO}_{2}$ pro $5 \mathrm{~g}$, entsprechend $1,0$ bis $0,013 \% \mathrm{C}$ (ccm $\times 0,0107)$.

Die Bestimmungen ergaben, nach dem $\mathrm{CO}_{2}$-Gehalt georänet :

\begin{tabular}{|c|c|c|c|}
\hline $\begin{array}{l}5 \mathrm{~g} \\
\mathrm{Nr} .\end{array}$ & $\begin{array}{l}\mathrm{CO}_{\mathrm{S}} \\
\mathrm{ccm} \\
\text { b. } 0^{\circ}\end{array}$ & $\begin{array}{c}\text { \% Asche } \\
\text { im Porzellantiegel } \\
\text { mit Bunsenbrenner }\end{array}$ & \\
\hline 1 & 93,20 & 18,50 & Kohle \\
\hline 2 & 39,82 & 62,50 & Waschberge \\
\hline$\overline{3}$ & 27,80 & 11,80 & Gaskohle \\
\hline 4 & 25,23 & 68,70 & Wasch berge \\
\hline 5 & 16,80 & 11,10 & Gaskohle \\
\hline 6 & 15,93 & 21,95 & Koble \\
\hline 7 & 7,55 & 6,60 & Kohle \\
\hline 8 & 6,71 & 10,60 & Gaskohle \\
\hline 9 & 6,06 & 16,87 & Koble \\
\hline 10 & 5,24 & 29,96 & Kohle \\
\hline 11 & 2,28 & 8,15 & Gaskohle \\
\hline 12 & 1,18 & 7,50 & Gaskohle \\
\hline
\end{tabular}

Aus den cem $\mathrm{CO}_{2}$ bei $0^{\circ}$ aus $5 \mathrm{~g}$ Kohle erhält man die Prozente $\mathrm{C}$ und $\mathrm{CO}_{2}$.

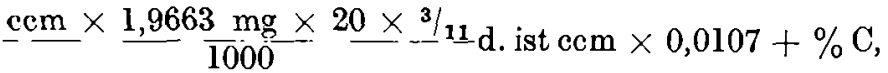

$$
\begin{aligned}
& \text { und } \underline{\mathrm{cm}} \times \frac{\times 1,9663 \times 20}{1000} \times \frac{2}{\mathrm{~d}} \text {. ist ccm } \times 0,04=\% \mathrm{CO}_{2} \text {. }
\end{aligned}
$$

So ist z. B. in Probe $1=93,20 \mathrm{ccm} \times 0,0107=1,00 \% \mathrm{C}$ und $93,20 \times 004=3,73 \% \mathrm{CO}_{2}$ und in Probe $2=39,82 \mathrm{ccm}$ $\times 0,0107=0,43 \% \mathrm{C}$ und $39,82 \times 0,04=1,60 \% \mathrm{CO}_{2}$

Als Beispiel für die Fehlerquelle aus dem $\mathrm{CO}_{2}$-Gehalt mag die Elementaranalyse der Probe $\Lambda^{\prime}$ r. I angeführt werden.

3) Für genaue Aschenbestimmungen sollten Platingeräte nicht verwendet werden, da das Gewicht des Platins je nach Reinheit beim längeren Glühen abnimmt.
Dieselbe ergab (Aschenbestimmung im Porzellantiegel mit dem Bunsenbrenner):

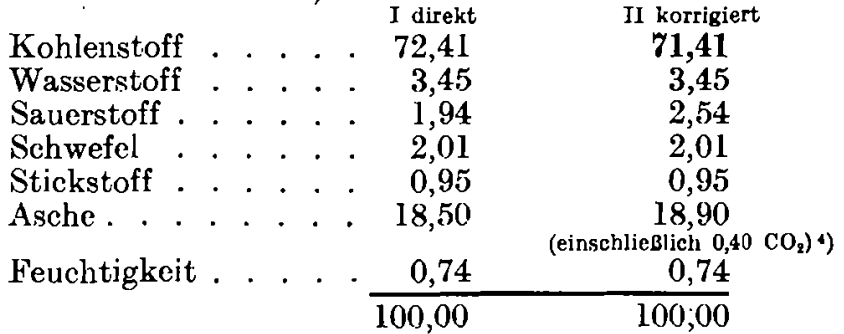

Die korrigierten Werte für $\mathrm{C}$ und $\mathrm{O}$ sind unter III aufgeführt. Da aus $5 \mathrm{~g}$ Kohle $93,2 \mathrm{ccm} \mathrm{CO}_{2}$ bei $0^{\circ}$ erhalten werden, so ist vom $\mathrm{C}=93,2 \times 0,0107 \mathrm{~d}$. i. $1,0 \% \mathrm{C}$ abzuziehen.

In diesem Falle war die Asche vor Berechnung des 0 nicht mit kohlensaurem Ammoniak behandelt. „Angenommen" es wären hier, wie bei der Aschen bestimmung in Kohle 3 (linke Spalte) auch $0,40 \% \mathrm{CO}_{2}$ entwichen, so ist von $\mathrm{O}=0,40$ minus $1,0 \mathrm{C}$ zu subtrahieren, $\mathrm{d}$. ist $0,60 \%$ zu addieren. Wie schon oben gesagt, kann man sich mit einer solchen ,Annahme" natürlich nicht begnügen, der Fehler kann bei dieser Kohle bis zu $(93,2 \times 0,04 \times 8 / 11)=2,71 \% 0$ betragen; die Korrektur muß nach den Angaben unter 3. vorgenommen werden.

Der Heizwert der Kohle berechnet sich:

ad I. (nicht korrigiert) $=6818^{\circ}$ WE. (Wasser als Dampf) ad II. (korrigiert) =: $6709 \mathrm{WE}$. (Wasser als Dampf) Differenz 109 WE.

Von dieser Differenz kommen 81 WE. auf den durch die Kohlensäure der Kohle zu hoch gefundenen C-Gehalt, und 28 WE. auf den um $0,60 \%$ zu niedrig berechneten 0 . Man ersieht schon hieraus, wie durch Nichtbeachtung der kohlensauren Frden bedeutende Fehler entstehen können.

Verfährt man nach 3., indem man v o r Berechnung des $\mathbf{O}$ aus der Differenz die Asche mit etwas kohlensaurem Ammoniak erhitzt, so besteht die ganze Korrektur darin, daß vom gefundenen $\mathrm{C}$ das der $\mathrm{CO}_{2}$ entsprechende Gewicht $\mathrm{C}$ subtrahiert und das gleiche Gewicht dem $\mathrm{O}$ addiert wird. Beträgt z. B. die $\mathrm{CO}_{2} 3,67 \%$ entsprechend $1,0 \% \mathrm{C}$, und ist durch Elementaranalyse gefunden $71,00 \% \mathrm{C}$ und $4,00 \% \mathrm{O}$ (als Differenz $\mathrm{n}$ a $\mathrm{c} \mathrm{h}$ dem Behandeln der Asche mit kohlensaurem Ammoniak), so sind die korrigierten Werte 70,00\% C und $\mathbf{5 , 0 0} \% \mathrm{O}$.

Verfährt man umgekehrt, indem man vor der $\mathrm{O}$-Berechnung alle $\mathrm{CO}_{2}$ aus der Asche austreibt, so ist vom $\mathrm{C}=3 / 11$ und vom $\mathrm{O}=8 / 11$ der $\mathrm{CO}_{2} \mathrm{zu}$ subtrahieren, somit $71,0 \mathrm{C}$ minus $(3,67 \times 3 / 11)==70,0 \% \mathrm{C}$ und $(4,00+3,67)$ minus $3,67 \times 8 / 11=5,00 \% 0$.

Die oben aufgeführten Kohlenproben kamen zur Untersuchung, wie dieselben gerade eingesandt wurden. Entstehen nun hier bei der geringen Zahl Proben (abgesehen von der Differenz bei der O-Bestimmung) beim (C schon Febler von $0,43 \%$ ( $\mathrm{Nr} .2$ ) und von $1,00 \%$ ( $\mathrm{Nr} . \mathrm{l}$ ), so ist ein noch größerer Einfluß durch die $\mathrm{CO}_{2}$ keineswegs ausgeschlossen. Bei der so einfachen und raschen Bestimmung in der unten beschriebenen Weise sollte man sich, wo der Aschengehalt nicht gering ist, Aufschluß über diesen Punkt verschaffen, auf jeden Fall aber dann, wenn es sich um den Heizwert handelt.

Es ist noch die Frage aufzuwerfen, ob die in der unten beschriebenen Weise bestimmte Kohlensäure auch an Kalk

4) Auch bei Berechnung der brennbaren Substanz ist in solchen Fälleu (Asche + entwichene $\mathrm{CO}_{2}$ ) einzusetzen, anderenfalls können größere Differenzen entstehen, so bei Probe 1 bis zu fast $3,73 \%$, bei Probe 2 bis fast $1,60 \%$ und bei Nr. 3 und 4 von ca. $1 \%$. Bei hoher Tenperatur erhält man bei Nr. 1 18,90-3,73 $=15,17 \%$ Asche, dieselbe Menge aber auch bei einer Kohle mit 15,67\% Asche, wenn $0,50^{\circ} ; \mathrm{CO}_{2}$ darin vorhanden sind. In beiden Fällen ist der Aschengehalt "scheinbur" gleich (ohne Korrektur für die entwichene Kohlensäure), differiert aber um $18,90-15,67=3,23 \%$, ein Fehler, der sich auf die brennbare Substanz überträgt. Wird bei niedrigerer Temperatur eingeäschert. so sind die Fehler ohne Korrektur von verschiedener Größe.

(Bei Berechnung des K. W. nach der $G$ o $u$ t a 1 schen Formol ist diese Korrektur auch zu berücksichtigen bezüglich der Größen von V.u. a.). 
( $\mathrm{Mg}$ ) gebunden ist und so bei der Elementaranalyse vollständig in dem Kaliapparat mit gewogen wird, oder ob und inwieweit das nicht der Fall ist beim Vorhandensein von kohlensauren Alkalien. Um dieses festzustellen, wurde die Asche von je 2,5 g der Proben 2, 3 und 5 noch im Verbrennungsrohr im Sauerstoffstrom erhitzt und in gleicher Weise auf Kohlensäure untersucht. Es wurden erhalten aus der Asche auf $5 \mathrm{~g}$ Kohle bezogen

$\begin{array}{cccc} & & \mathrm{CO}_{2} \text { b. } 00 & \begin{array}{c}\text { eutsprechend } \\ \% \mathrm{C}\end{array} \\ \text { Probe Nr. } 2 & 2,60 \mathrm{ccm} & 0,028 \\ \text { " " } & 3 & 1,83 ., & 0,020 \\ \text { " } & 5 & \mathbf{0 , 5 4}, " & 0,006\end{array}$

Es ist dies somit gegen die Kohlensäure aus diesen Kohlen direkt $(39,82,27,80$ und $16,80 \mathrm{ccm})$ sehr gering, und die Resultate werden hier nur um 0,028 bis $0,006 \% \mathrm{C}$ dadurch niedriger, da $\mathrm{B}$ die $\mathrm{CO}_{2}$, welche bei der Elementaranalyse in der Asche bleibt, als C mit subtrahiert wird; von der Bestimmung der an Alkalien gebundenen $\mathrm{CO}_{2}$, welche in derim Rohre ausgeglühten Asche zurückbleibt, kann man somit bei $\mathrm{S}$ t e i $\mathrm{n}$ kohle absehen.

Für die bei der Verbrennung eintretende Veränderung der Asche durch den S- und N-Gehalt der Kohle ist nicht so leicht auch eine zutreffende Korrektur einzusetzen. Soweit der Schwefel nicht in flüchtiger Form ausgetrieben wird, oder als schwefelsaures Salz vorhanden ist, entweicht für die gebildete Schwefelsäure $\mathrm{CO}_{2}$, so daß das Gewicht praktisch gleich bleibt $\left(480: 44 \mathrm{CO}_{2}\right)$. Dies gilt auch von der aus dem Schwefelkics entsteherden $\mathrm{SO}_{3}$, während das gebildete $\mathrm{Fe}_{2} \mathrm{O}_{3}$ gegen das $\mathrm{Fe}_{2}$ die Asche etwas verringert und den $\mathrm{O}$ entsprechend erhölt; da aber nur cin Achtel hiervon auf den disponiblen $H$ entfällt, so kann der Einfluß des $\mathrm{S}$ vernachlässigt werclen, ebenso der des $\mathrm{N}$, da es sich hicrmit ähnlich verbält. Aber ohne Zweifel wird die Korrektur für die $\mathrm{CO}_{2}$ nicht selten zu einer genaueren Elementaranalyse oder Heizwertbestimmung führen.

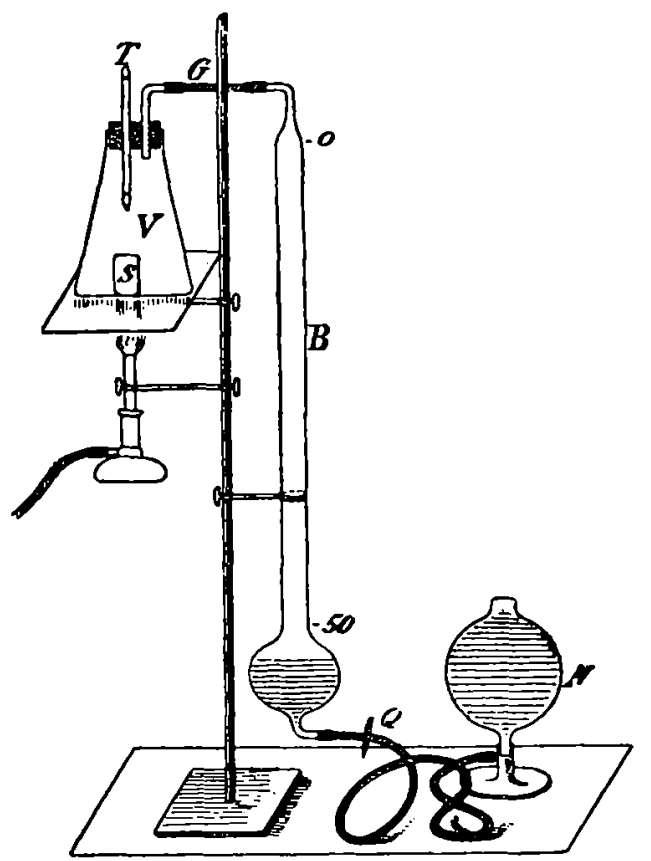

Die Bestimmung der $\mathrm{CO}_{2}$ führe ich bei diesen Substanzen in der abgebildeten Vorrichtung aus, wobei auf möglichste Einfachheit und rasche Ausführung Rücksicht genommen ist. Indessen lassen die Resultate für diesen Zweck nichts zu wünschen übrig. Wer es vorzieht, kann ja auch die $\mathrm{CO}_{2}$ nach anderen Methoden bestimmen.

Der rechtwinklige Ansatz an der Bürette $B$ ist durch ein Stück Schlauch $G$ (ca. $10 \mathrm{~cm}$ ) mit dem oben weiten Gefäß $V$ verbunden, damit man dieses leicht bewegen kann. Man gibt $5 \mathrm{~g}$ fein zerriebene Kohle und $5 \mathrm{ccm}$ Wasser in $V$ und bringt dann das bis etwa zur Hälfte mit $5 \mathrm{ccm}$ Salzsäure angefüllte Gli chen $S$ (gleiche Teile konz. HCl und Wasser) mit ciner Pinzette auf den Boden von V. Man stellt das Wasser in $B$ auf annähernd 0 ein, setzt den mit einem Thermometer $T$ versehenen

Gummistopfen in $V$ ein, wartet kurze Zeit, bis die Tempera tur konstant geworden ist, und gleicht dann mit der Niveauflasche den Druck aus. Nachdem der Stand in der Büretto und die Temperatur notiert ist, neigt man $V$, so daß die Säure durch Umkippen von $S$ entleert wird, und crwärmt $V$ nach und nach zum Kochen bei dauernd g e of f nete $\mathrm{m}$ Quetschhahn $Q$, indem man $N$ mit dem Sinken des Wassers in $B$ nach und nach niedriger stellt. Man erhitze nicht zu rasch und koche nicht zu lebhaft, damit das Kohlenpulver nicht zu hoch steigt, und der Wasserdampf vor Austritt aus $V$ kondensiert wird; durch Umschwenken von $V$ kann man die Kohle von der Wandung abspülen. Die Bürette ist von 0 bis $50 \mathrm{ccm}$ eingeteilt, unten aber mit einer Kugel von 100 bis $150 \mathrm{ccm}$ Fassungsraum versehen, weil durch die hohe Temperatur und den Wasserdampf in $V$ das Gasvolumen auch bei wenig $\mathrm{CO}_{2}$ zunächst recht groß wird.

Nachdem etwas abgekühlt ist, stellt man $V$ in kaltes Wasser, indem man $N$ bei geöffnetem Quetschhahn nach und nach hebt; sobald die Anfangstemperatur wieder genau erreicht ist, liest man den Stand der Bürette ab. Es braucht kaum erwäbnt zu werden, daß die Versuche an einem vor Temperaturwechsel geschützten Orte auszuführen sind; nach der nötigen Kühlung von $V$ ist dann auch in $B$ die Anfangstemperatur wieder eingetreten. Kleine Temperaturdifferenzen sind hier nicht von Bedeutung, da $1 \mathrm{ccm}$ nur $0,0107 \% \mathrm{C}$ entspricht, ein Wassermantel um $B$ ist daher nicht nötig; Barometerstand und Tension können hier unberücksichtigt bleiben.

So ergaben z. B. $5 \mathrm{~g}$ der Probe Nr. 2

Stand der Bürette nach aufgesetztem Stopfen nach dem Versuch und Abkühlen auf $16^{\circ}$

$=1,80 \mathrm{ccm}$ bej $16^{\circ}$

$=44,10 \mathrm{ccm}$

$=42,30 \mathrm{ccm} \mathrm{CO}_{2}$ bei $16^{\circ}$

entsprechend $=39,82 \mathrm{ccm} \mathrm{CO}_{2}$ bei $0^{\circ} \times 0,0107=0,43 \% \mathrm{C}$.

Sollte bei dem Versuche mehr $\mathrm{CO}_{2}$ als bis zur Marke $50 \mathrm{ccm}$ erhalten werden, so ist derselbe mit weniger Substanz zu wiederholen.

Bei KohleNr. I wurden

aus $2,5 \mathrm{~g}$ erhalten $=49,5 \mathrm{ccm}$ bei $16^{\circ}$

entsprechend aus $5 \mathrm{~g}$

erhalten

$=99,0 \mathrm{ccm}$ bei $16^{\circ}$

$=93,2 \mathrm{ccm}$ bei $0^{\circ} \times 0,0107=1,00 \% \mathrm{C}$.

Man könnte auch eine Bürette von $100 \mathrm{ccm}$ wählen, dic Vorrichtung ist dann aber bei längerer Bürette nicht so handlich, und bei weiterer Bürette ist das Resultat weniger genau.

Es kommt vor, aber sehr selten, daß dic entwickelte $\mathrm{CO}_{2}$ eine geringe Menge Schwefelwasserstoff enthält. Bei dem großen Luftüberschuß ist nach Beendigung des Vorsuches nur noch ein Teil davon vorhanden. Es lohnt sich somit nicht, den Apparat für die Trennung der beiden Gase viel komplizierter zu gestalten.

[A. 121.]

\section{Bestimmung des Broms und Jods in Gegenwart von Chloriden.}

Von L. W. IVINGLER, Budapest.

(SchluB von S. 480.)

Bezüglich cier Jodbestimmung sollen hier folgende Angaben Platz finden

1. Am einfachsten läßt sich in einem löslichen Jodid die Menge des Jods bestimmen, wenn man zur angesäuerten Lösung etwas Nitritlösung hinzufügt, dann für das Unschädlichmachen des überschüssigen Nitrits mit Verwendung von $\mathrm{H}$ a $r \mathrm{n} s \mathrm{t}$ of $\mathrm{f}$ sorgt, endlich das ausgeschiedene Jod in üblicher Weise mißt.

Die Ausführung des Verfahrens gestaltet sich wie folgt: Man gibt in eine Glasstöpselflasche von etwa $150 \mathrm{ccm}$ Inhalt $50 \mathrm{ccm}$ von der Jodidlösung, fügt $25 \mathrm{ccm}$ Tetrachlorkohlenstoff hinzu, säuert die Flüssigkeit mit $0,5 \mathrm{ccm}$ (10 Tropfen) $10 \%$ iger Sulzsäure an und träufelt zuletzt 2 Tropfen Natriumnitritlösung $(1 \%)$ in die Flasche. Man läßt nun unter öfterem Durchschütteln eine halbe Stunde stehen, streut dann in die Flasche $1 \mathrm{~g}$ reinsten Harnstoff, schüttelt wieder 